\title{
Thermal stability of a special class of black hole solutions in $F(R)$ gravity
}

\author{
S. H. Hendi ${ }^{1,2, a} \mathbb{C}$, R. Ramezani-Arani ${ }^{3}$, E. Rahimi ${ }^{3}$ \\ ${ }^{1}$ Physics Department and Biruni Observatory, College of Sciences, Shiraz University, Shiraz 71454, Iran \\ ${ }^{2}$ Research Institute for Astronomy and Astrophysics of Maragha (RIAAM), P.O. Box 55134-441, Maragha, Iran \\ ${ }^{3}$ Department of Elementary Particles, Faculty of Physics, University of Kashan, Kashan, Islamic Republic of Iran
}

Received: 24 July 2018 / Accepted: 22 May 2019 / Published online: 4 June 2019

(C) The Author(s) 2019

\begin{abstract}
In this paper, we work on the topological Lifshitzlike black hole solutions of a special class of vacuum $F(R)$-gravity that are static and spherically symmetric. We investigate geometric and thermodynamic properties of the solutions with due respect to the validity of the first law of thermodynamics. We examine the van der Waals like behavior for asymptotically AdS solutions with spherical horizon by studying the $P-v, G-T$ and $C_{Q, P}-r_{+}$diagrams and find a consistent result. We also investigate the same behavior for hyperbolic horizon and interestingly find that the system under study can experience a phase transition with negative temperature.
\end{abstract}

\section{Introduction}

$F(R)$ gravity is one of the best models of modified general relativity with a renewed interest in recent years. In addition to the simple and general Lagrangian of this model, the main (but not the first) motivation of considering $F(R)$ gravity with arbitrary function of Ricci scalar is that one can explain the accelerated expansion and structure formation of the Universe without considering dark energy or dark matter. It is also believed that some curvature corrections arising from quantum theory of gravity may be collected to special functional forms of $F(R)$ gravity.

In other words, some defects in Einstein's general relativity and the motivation of studying more complete and general gravitational models, have led to the creation of general modified gravity models. These gravitational models have been considered in various branches of gravity, cosmology, astrophysics and their interesting results have made these models appropriate generalization for the Einstein's gravity [1-3]. In the lastest decade, special attention was given to modified

\footnotetext{
a e-mail: hendi@shirazu.ac.ir
}

theories that generalize the gravitational action integral with the simplest model, the so-called $F(R)$ gravity [4]. There are two approaches for obtaining the field equations of the generalized $F(R)$ gravity. The first one is the standard metric formalism and the other one is the Palatini method [5] in which metric tensor and affine connection are treated, a priori, as independent variables. There are many viable $F(R)$ models that can satisfy both cosmological and local gravity constraints [6-9]. Moreover, $F(R)$ gravity can be reduced to general relativity in the specific cases [10]. Nowadays, $F(R)$ gravity has attracted much attentions of the researchers and there are many studies in this field [11-14].

The generalization of $F(R)$ theory in the context of Horava-Lifshitz gravity and its interesting cosmological results have been addressed in series of papers [15-24]. The pioneer work of Horava in 2009 [25], the so-called HoravaLifshitz gravity is one of the candidate theories of quantum gravity. Since general relativity is not a renormalizable theory (it means that it is successful as a classical theory of gravity, but it breaks down at some scale), and therefore, it should be viewed as an effective theory. Beyond that scale, general relativity is not suitable theory to describe the gravitational interactions or spacetime itself and one cannot construct its quantum counterpart using conventional quantization techniques.

On the other hand, within this perspective that general relativity is an effective theory, it may solve some problems in gravitation by treating the quantum concept more fundamentally, and so, space and time are not equivalent (anisotropic) at high energy level. The relativistic concept of time with its Lorentz invariance emerges at large distances. The theory relies on the theory of foliations to produce its causal structure. It is related to topologically massive gravity and the Cotton tensor. So it may have a possible UV completion of general relativity to address this issue. Recently, the proposed Horava-Lifshitz gravity promises a UV completion of 
Einstein's theory by sacrificing general covariance at short distances and introducing anisotropic spacetime scaling. In addition, Donoghue showed that general relativity and quantum field theory can be perfectly compatible if the quantum gravity is formulated as an effective field theory [26]. Therefore, the effective field theory of quantum gravity is valid above which the effective description is replaced with the UV completion. Furthermore, Horava-Lifshitz gravity theory can be employed as a covariant framework to build an effective field theory for the fractional quantum Hall effect that respects all the spacetime symmetries such as non-relativistic diffeomorphism invariance and anisotropic Weyl invariance as well as the gauge symmetry. Consequently, investigation of Horava-Lifshitz gravity family can help us to deepen our insight for moving from classical gravity point of view to quantum one.

According to the discovery of Hawking radiation [27], one finds that the black hole thermodynamics has crucial role for studying the quantum nature of gravity. Hawking tried to show that black holes behave like black bodies in the usual thermodynamic sense, emitting radiation with a thermal spectrum $[27,28]$. In this regard, many authors have tried to obtain a new approach to find a statistical origin to Bekenstein-Hawking entropy [29-31].

The entropy of Einsteinian black hole, known as the Bekenstein-Hawking area law entropy, suggests that the quantum degrees of freedom of a typical black hole are effectively distributed over a surface, rather than a volume. Following the works of 't Hooft [32] and Susskind [33], one can find this crucial result based on the holographic principle, in which says that quantum gravity in a given volume should be described by a theory on the boundary of that volume. The mentioned holographic principle is related to the so-called anti-de Sitter/conformal field theory (AdS/CFT) correspondence $[34,35]$. In the other words, in order to explain the holographic principle, a consistent quantum gravity theory should admit two equivalent descriptions (AdS/CFT correspondence): one as a bulk semiclassical general relativity theory, and one as a boundary quantum field theory. So, investigation of black hole solutions with AdS asymptote, which has an undeniable role for constructing a consistent quantum gravity theory [36], has special interest.

Recently, a renewed interest in phase transition of asymptotically AdS black holes, especially van der Waals like [37,38], has appeared. Subsequently, a number of interesting results, such as, triple points [39-41], reentrant phase transitions [42-44], and analogous Carnot-cycle heat engines [4547] were obtained. Regardless of massive gravity black holes [48], it is known that the van der Waals behavior in Einstein gravity is seen only for AdS black holes with spherical horizon [37], and therefore, such behavior does not take place for AdS black holes with flat or hyperbolic horizons (no real critical point is found). In this paper, we observe that in addition to spherical horizon, one can find the van der Waals behavior in the especial case of $F(R)$ gravity with hyperbolic horizon. It is note that in this case, although the critical volume and pressure are positive, the critical temperature is negative. Since negative temperature has a physical interpretation in usual thermodynamics of quantum system (e.g: a system of nuclear spins in an external magnetic field or population inversion in laser [49-53], it will be interesting to investigate our solutions with quantum mechanical point of view.

Thermodynamic descriptions of gravitational solutions at the event horizon and cosmological ones at the apparent horizon in $F(R)$ gravity have been studied in series of papers [54-62] . In this work, we are going to evaluate thermodynamic phase transition of black hole solutions in a special class of $F(R)$ gravity with constant Ricci scalar in which both $F(R)$ and its derivative $F_{R}(R)$ are vanished in the field equations. The structure of our paper is as follows: in next section, we are going to introduce $F(R)$ gravity, in brief, and then we obtain the special class of exact solutions with black hole interpretation. Then we investigate geometric and thermodynamic properties. In Sec. 3, we work in the extended phase space and investigate the possible phase transition and van der Waals like behavior. We also discuss thermal stability and critical quantities. Final section is devoted to some concluding remarks.

\section{Basic equations and dynamic black hole solutions}

The purpose of this paper is to study the thermodynamics of a typical anti-de Sitter black hole solution in four dimensional $F(R)=R+f(R)$ gravity in which the Ricci scalar is constant $\left(R=R_{0}\right)$. Let us first consider the 4-dimensional action of $R+f(R)$ gravity which is given by

$S=\int_{\mathcal{M}} d^{4} x \sqrt{-g} F(R)=\int_{\mathcal{M}} d^{4} x \sqrt{-g}[R+f(R)]$,

where $\mathcal{M}$ is a four-dimensional bulk manifold. It is clear that for $F(R)=R(f(R)=0)$, one can recover the HilbertEinstein action of General Relativity. Using the variational principle on the action of $F(R)$ gravity (1), it is a matter of calculation to show that the field equation is given by

$R_{\mu \nu}\left[1+f_{R}\right]-\frac{1}{2}[R+f(R)] g_{\mu \nu}+\left[g_{\mu \nu} \square-\nabla_{\mu} \nabla_{\nu}\right] f_{R}=0$,

where we use the notation $A_{B}=\frac{d A}{d B}$. It is easy to show that one can rewrite Eq. (2) with the following form

$G_{\mu \nu} F_{R}=\frac{1}{2} g_{\mu \nu}\left[F(R)-R F_{R}\right]+\left[\nabla_{\mu} \nabla_{\nu}-g_{\mu \nu} \square\right] F_{R}$. 
In this paper, we follow the method of Ref. [63], which is a special class of $F(R)$-gravity models with two constraints, simultaneously, $F\left(R_{0}\right)=0$ and $F_{R}=0$. Taking into account the mentioned constraints, one can find that the vacuum equation (3) are automatically satisfied with arbitrary $R_{0}$. It is notable that the mentioned class does not cover the usual general relativity solutions since the vacuum field equation of general relativity with arbitrary metric identically satisfied $F_{R}=1$ with vanishing Ricci scalar. Consequently, our strategy is working on some viable models of $F(R)$-gravity that satisfy these conditions and solving the equation of constant Ricci scalar. As it is mentioned in [63], there are several models for the early-time inflation or late-time accelerated expansion that can satisfy the mentioned constraints. As a result, we regard a spherically symmetric and static solutions with constant Ricci scalar which are reported in [63] to investigate their possible phase transition.

Here, our main motivation is the study of thermodynamical and geometrical aspects of topological black hole solutions with Lifshitz-like spacetime. Therefore, we consider the metric of 4-dimensional spacetime as [63]

$d s^{2}=-e^{2 \alpha(r)} B(r) d t^{2}+\frac{d r^{2}}{B(r)}+r^{2} d \Omega^{2}$,

in which

$d \Omega_{k}^{2}=\left\{\begin{array}{cc}d \theta^{2}+\sin ^{2} \theta d \varphi^{2} & k=1 \\ d \theta^{2}+d \varphi^{2} & k=0 \\ d \theta^{2}+\sinh ^{2} \theta d \varphi^{2} & k=-1\end{array}\right.$,

where $k=1,0$ and -1 represent spherical, flat and hyperbolic horizon of possible black holes, respectively. Hereafter, we indicate $\omega_{k}$ as the volume of boundary $t=$ cte and $r=c t e$ of the metric. Since we desire to study the Lifshitzlike solutions [63], we define $\alpha(r)$ as

$\alpha(r)=\frac{1}{2} \ln \left(\frac{r}{r_{0}}\right)^{z}$

where $z$ is a real number and $r_{0}$ is an arbitrary (positive) length scale. It is notable that in order to obtain a dimensionless argument of logarithmic function, the existence of $r_{0}$ is necessary. Inserting Eq. (6) in the introduced metric (4), one can obtain

$d s^{2}=-\left(\frac{r}{r_{0}}\right)^{z} B(r) d t^{2}+\frac{d r^{2}}{B(r)}+r^{2} d \Omega^{2}$.

As an additional comment, we note that although we can start with Eq. (7) as the line element, it is convenient to define Eq. (4 ) at the first step to guarantee that the Lifshitz factor $\left(e^{2 \alpha(r)}\right)$ is positive definite and change of signature of the metric comes from the sign of $B(r)$ (the number of plus (+) and minus (-) signs is unchanged). Considering the metric (7) with the mentioned field equation (2) (and also the mentioned constraints for special class of $F(R)$-gravity), one can extract the metric function for $R=R_{0}$, where

$R=R_{0}=-\frac{d^{2} B(r)}{d r^{2}}-\frac{3 z+8}{2 r} \frac{d B(r)}{d r}-\frac{z^{2}+2 z+4}{2 r^{2}} B(r)+\frac{2 k}{r^{2}}$,

with the following exact solutions [63]

$B(r)=K-\frac{C_{ \pm}}{r^{b_{ \pm}}}-\lambda r^{2}$,

where $K$ and $\lambda$, are two (positive/negative or zero) constants which their values are depending on the signs/values of $k$ and $R_{0}$ as

$K=\frac{4 k}{z^{2}+2 z+4}$,
$\lambda=\frac{2 R_{0}}{z^{2}+8 z+24}$.

In addition, $C_{ \pm}$are two integration constants while $b_{ \pm}$is

$b_{ \pm}=\frac{1}{4}\left(3 z+6 \pm \sqrt{z^{2}+20 z+4}\right)$.

Avoiding complex values of $B(r)$, one may regard the following constrain on $z$

$$
\begin{aligned}
& z^{2}+20 z+4>0 \\
& \quad \text { or equivalently } z \notin[-2(2 \sqrt{6}+5), 2(2 \sqrt{6}-5)] .
\end{aligned}
$$

According to the above constrain on $z$, one can find the allowed ranges of $b_{ \pm}$as follows

$b_{-} \in(-\infty,-13.35] \quad$ when $\quad z<-2(2 \sqrt{6}+5)$,

$b_{-} \in[0.93,+\infty) \quad$ when $\quad z>2(2 \sqrt{6}-5)$.

and

$b_{+} \in(-\infty,-12.93] \quad$ when $\quad z<-2(5+2 \sqrt{6})$,

$b_{+} \in[1.35,+\infty) \quad$ when $\quad z>2(2 \sqrt{6}-5)$.

In order to interpret the solutions as black holes, we should examine the existence of horizon and singularity for the singular black holes. The presence of singularity could be investigated by studying curvature scalars for which we choose the 
Kretschmann scalar. It is a matter of calculation to show that for these solutions, the Kretschmann scalar is

$$
\begin{aligned}
R_{\alpha \beta \gamma \delta} R^{\alpha \beta \gamma \delta}= & \left(\frac{d^{2} B(r)}{d r^{2}}\right)^{2} \\
& +\left(\frac{z\left(3 \frac{d B(r)}{d r}+z B(r)-2 B(r)\right)}{r^{2}}\right) \frac{d^{2} B(r)}{d r^{2}} \\
& +\frac{9 z^{2}+16}{4 r^{2}}\left(\frac{d B(r)}{d r}\right)^{2} \\
& +\left(\frac{z\left(3 z^{2}-6 z+8\right) B(r)}{2 r^{3}}\right) \frac{d B(r)}{d r} \\
& +\frac{z^{4}-4 z^{3}+12 z^{2}+16}{4 r^{4}} B^{2}(r) \\
& -\frac{8 k}{r^{4}} B(r)+\frac{4 k^{2}}{r^{4}} .
\end{aligned}
$$

It is straightforward to show that Eq. (18) diverges at $r=0$ and it is finite for $r \neq 0$. In addition, according to the Fig. 1, one finds that the metric function has at least one real positive root (with positive slope). As a result, the mentioned solutions can be interpreted as black holes. In addition, according to Fig. 1 and also Eq. (9), one finds that for positive and negative $R_{0}$, one can find asymptotically dS and adS, respectively with an effective cosmological constant, $\Lambda_{e f f}=\frac{6 R_{0}}{z^{2}+8 z+24}$. Moreover, it is clear that for vanishing $R_{0}$ and $k=1$ the mentioned solutions are asymptotically flat only for $z=0$ [we should note that although $z=-2$ with $k=1$ leads to $K=1$, but $z=-2$ is not allowed based on Eq. (13)].

Now, we are in a position to study thermodynamical properties of the solutions and investigate their thermal stability based on the heat capacity.

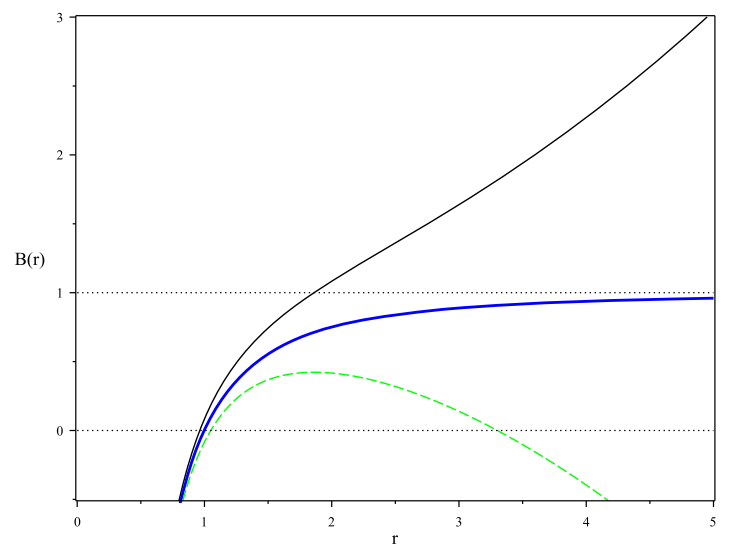

\subsection{Conserved and thermodynamical quantities}

Here, we are going to calculate the conserved and thermodynamic quantities of black holes in $F(R)$ gravity. Due to the fact that the employed metric contains a temporal Killing vector, we use the concept of surface gravity to calculate the temperature of black holes at the event horizon $r_{+}$

$$
T=\frac{1}{2 \pi} \sqrt{\frac{1}{2}\left(\nabla_{\mu} \chi_{\nu}\right)\left(\nabla^{\mu} \chi^{\nu}\right)}=\left.\frac{B^{\prime}(r)}{4 \pi}\left(\frac{r}{r_{0}}\right)^{\frac{z}{2}}\right|_{r=r_{+}},
$$

where $\chi^{v}=\delta_{0}^{v}$ is the Killing vector. Regarding Eq. (19) with metric function (9), one can find

$$
T=\frac{\left[\left(K-\lambda r_{+}^{2}\right) b_{-}-2 \lambda r_{+}^{2}\right] r_{+}^{b_{+}}-C_{+}\left(b_{+}-b_{-}\right)}{4 \pi r_{+}^{1+b_{+}}}\left(\frac{r_{+}}{r_{0}}\right)^{\frac{z}{2}},
$$

where $C_{+}$is removed due to the fact that the metric function vanishes on the event horizon, $r_{+}$.

In order to study the entropy of black holes in $F(R)=$ $R+f(R)$ gravity, one can use the generalized area law [1]. According to the result of Ref. [1], the entropy can be calculated as

$S=\frac{A}{4}\left[1+f_{R}\left(R_{0}\right)\right]$

where $A$ is the event horizon area of the black holes. But here we have used two constraints $F(R)=0$ and $F_{R}=0$, and therefore, Eq. (21) leads to zero entropy. This problem comes from the fact that the entropy of $F(R)$ gravity (in the non-equilibrium description of thermodynamics [64]) is a modification of the area law with an effective gravitational coupling $\left(G_{e f f}=G / F_{R}\right)$. Since in our case we have $F_{R}=0$ and the effective gravitational coupling diverges, we could

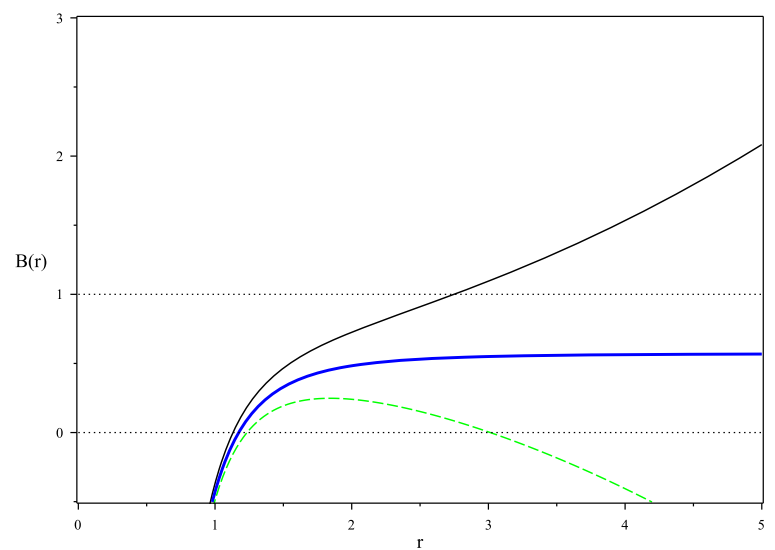

Fig. $1 \mathrm{~B}(\mathrm{r})$ versus $r$ for $C_{+}=1, C_{-}=0, k=1$, and $R_{0}=-1$ (black-continuous line), $R_{0}=0$ (blue-bold line) and $R_{0}=1$ (green-dashed line). "left figure: $z=0$ and right figure: $z=1$ " 
not use the mentioned modified area law relation. In other words, the usual Wald approach is break down and we have to use an alternative method. Since we believe that the black hole solutions should satisfy the laws of thermodynamics, we use the first law to calculate the nonzero entropy.

Taking into account the timelike Killing vector $(\partial / \partial t)$, one can show that the finite mass per unit volume $\omega_{k}$ can be obtained as

$M=\frac{1}{2}\left(K r_{+}^{b_{-}}-\lambda r_{+}^{2+b_{-}}-\frac{C_{+}}{r_{+}^{b_{+}-b_{-}}}\right)$.

Here, we desire to calculate the entropy in by using the validity of the first law of thermodynamics. It is easy to show that

$\delta M=T \delta S$,

and therefore, it is a matter of calculation to show that the following equality holds

$S=\int \frac{d M}{T}=\frac{4 \pi r_{+}^{1+b_{-}}}{2-z+2 b_{-}}\left(\frac{r_{0}}{r_{+}}\right)^{\frac{z}{2}}$.

It is notable that the obtained relation for the entropy reduces to the area law for $z=0\left(b_{-}=1\right)$. In other words, it seems that the $F(R)$ gravity does not direct effect on the entropy relation, such as that occurs in the equilibrium description of thermodynamics in $F(R)$ gravity [64].

\section{Extended phase space thermodynamics, thermal stability and phase transition}

Regarding the variation of the cosmological constant as the vacuum expectation value of a quantum field, one may expect to consider it and its conjugate in the first law of thermodynamics [65-67]. In this regard, the cosmological constant interpreted as a dynamical pressure of the black hole system as [37] (note: $\lambda \approx \frac{\Lambda}{3}$ )

$P=-\frac{3 \lambda}{8 \pi}$

where its conjugate extensive quantity is the thermodynamic volume which can be obtained by

$V=\left(\frac{\partial H}{\partial P}\right)_{S, C_{+}}$

in which $H$ is the enthalpy of system. We should note that in the extended phase space the mass of black hole is not the internal energy of the system, but its enthalpy $H \equiv M$. As an additional comment, it is worthwhile to mention that the modified Smarr relation which can be calculated by the scaling argument for our Lifshitz like solutions in the extended phase space is

$M=\frac{\left(2 b_{-}+2-z\right)}{2 b_{-}} T S-\frac{2}{b_{-}} P V+\frac{b_{+}}{b_{-}} \mathcal{C} C_{+}$,

where $\mathcal{C}=\left(\frac{\partial M}{\partial C_{+}}\right)_{S, P}=\frac{-1}{2 r_{+}^{b_{+}-b_{-}}}$can be interpreted as a modified potential per unit charge that calculated at the event horizon of Lifshitz like black hole solutions. One can confirm that Eq. (27) is reduced to that of charged AdS black holes for $z=0$. It is also notable that in the extended phase space, the modified first law of thermodynamics is completely in agreement with the modified Smarr relation and can be written as

$d M=T d S+V d P+\mathcal{C} d C_{+}$.

Moreover, we can calculate the modified volume in the Lifshitz like spacetime as

$V=\left(\frac{\partial M}{\partial P}\right)_{S, C_{+}}=\frac{4 \pi}{3} r_{+}^{2+b_{-}}$

where we see that the Lifshitz parameter modify the thermodynamic volume and for $z=0$ one can recover the usual volume $V=\frac{4 \pi r_{+}^{3}}{3}$.

Hereafter, since we desire to investigate the possible van der Waals phase transition, we regard negative cosmological constant (positive $P$ ) and spherical horizon $\left(S^{2}\right)$ with volume $\omega_{1}=4 \pi$. Regarding the relation for the temperature (20) with the mentioned equation of pressure (25), we can obtain the equation of state $P=P\left(T, r_{+}\right)$, as

$P=\frac{12 \pi r_{+}\left(\frac{r_{+}}{r_{0}}\right)^{-\frac{z}{2}} T-3 C_{+}\left(b_{+}-b_{-}\right) r_{+}^{-b_{+}}-3 K b_{+}}{8 \pi r_{+}^{2}\left(2+b_{+}\right)}$

where $r_{+}$is a function of the thermodynamic volume as indicated in Eq. (29). Following Ref. [37], one can find a relation between the horizon radius and specific volume. In the geometric units, we can obtain

$r_{+}=\frac{v}{2}$

As a result, we can work with specific volume, or directly, the radius of event horizon as volume representative.

Supposing the existence of critical behavior of the system in the $P-V$ isotherm diagram, one finds that the inflection point of $P-V$ diagram can be interpreted as the critical point on the critical isotherm with the following properties 
$\left(\frac{\partial P}{\partial r_{+}}\right)_{T}=\left(\frac{\partial^{2} P}{\partial r_{+}^{2}}\right)_{T}=0$

Using Eq. (32) and the equation of state (30), we can calculate the critical parameters as

$T_{c}=\frac{2 K b_{+} b_{-} \xi^{\frac{2-z}{2 b_{+}}}}{\pi(z+2)\left(2 b_{+}+2-z\right) r_{0}^{\frac{z}{2}}}$,

$r_{c}=\frac{v_{c}}{2}=\xi^{\frac{-1}{b_{+}}}$,

$P_{c}=-\frac{3 K(z-2) b_{-} b_{+}}{8 \pi(2+z)\left(2+b_{-}\right)\left(2+b_{+}\right)} \xi^{\frac{2}{b_{+}}}$,

These relations lead us to obtain the following ratio

$\rho_{c}=\frac{P_{c} v_{c}}{T_{c}}=\frac{-3(z-2)\left(2 b_{+}+2-z\right) r_{0}^{\frac{z}{2}}}{8\left(2+b_{-}\right)\left(2+b_{+}\right)} \xi^{\frac{z}{2 b_{+}}}$

where the constant $\xi$ is defined as

$\xi=\frac{2 K(z-2) b_{-}}{\left(2 b_{+}+2-z\right)\left(2+b_{+}\right)\left(b_{+}-b_{-}\right) C_{+}}$.

It is easy to check that for $z=0$, the mentioned ratio reduces to the van der Waals fluid, $\frac{3}{8}$.

On the other hand, thermodynamic behavior of a system can be investigated by some thermodynamic potentials such as the free energy. It is known that the enthalpy of black holes in the extended phase space is the total mass. The reason is due to the fact that the cosmological constant is no longer a fixed parameter but a thermodynamical one. Due to modification in interpretation of the total mass of the black holes in extended phase space, the Gibbs free energy is given by

$G=M-T S$, diagrams. The presence of both swallow-tail characteristic in $G-T$ diagrams and the inflection point in $P-r_{+}$diagrams indicate that our system undergoes a first order phase transition. Generally speaking, the van der Waals like phase transition between two different phases is characterized by a swallow-tail shape in $G-T$ diagrams and the inflection point in $P-r_{+}$plots. In our case, such a behavior represents a phase transition between small and large black holes.

Now, we desire to investigate thermal stability of the obtained black hole solutions. In order to discuss thermal stability of a black hole, one can calculate the heat capacity and discuss its sign (positivity/negativity) through its roots and divergencies. Basically, the conditions regarding thermal stability of black holes could be attained by studying the sign of heat capacity. Regardless of the values of parameters in the theory, positivity of the heat capacity ensures thermal stability of the solutions, whereas its negativity is considered to be an unstable state. Another advantage of investigating the heat capacity is the relation of its divergencies with phase-transition interpretation. Since we are working in the extended phase space, the heat capacity is given by

$C_{Q, P}=T\left(\frac{\partial S}{\partial T}\right)_{Q, P}=T \frac{\left(\frac{\partial S}{\partial r_{+}}\right)_{Q, P}}{\left(\frac{\partial T}{\partial r_{+}}\right)_{Q, P}}$

By using the equations of temperature (20) and entropy (24), we can obtain the heat capacity of black holes as

$C_{Q, P}=\frac{4 \pi r_{+}^{1+b_{-}} \chi}{\left(\frac{r_{+}}{r_{0}}\right)^{\frac{z}{2}}}$

where

$\chi=\frac{8 \pi P\left(2+b_{-}\right) r_{+}^{2+b_{+}}+3 K b_{-} r_{+}^{b_{+}}-3 C_{+}\left(b_{-}-b_{+}\right)}{8 \pi P\left(2+b_{-}\right)(z+2) r_{+}^{2+b_{+}}+3 K b_{-}(z-2) r_{+}^{b_{+}}-3 C_{+}\left(b_{-}-b_{+}\right)\left(z-2 b_{+}-2\right)}$

or equivalently its value per unit volume $\omega_{k}$ is

$$
\begin{aligned}
G= & \frac{K}{2} r_{+}^{b_{-}}\left(1-\frac{b_{+}}{1+b_{-}-\frac{z}{2}}\right) \\
& +\frac{4}{3} \pi \operatorname{Pr}_{+}^{2+b_{-}}\left(1-\frac{2+b_{+}}{1+b_{-}-\frac{z}{2}}\right) \\
& \left.-\frac{C_{+}}{2 r_{+}^{b_{+}-b_{-}}}\left(1+\frac{b_{+}-b_{-}}{1+b_{-}-\frac{z}{2}}\right)\right] .
\end{aligned}
$$

In order to investigate thermal behavior of the obtained black holes, we have plotted $P-r_{+}$isotherms and $G-T$

\subsection{Results and discussion}

Regarding Figs. 2, 3 and 4, one can find that for $T<T_{c}$ (in $P-r_{+}$diagrams) or $P<P_{c}$ (in $G-T$ and $C_{Q, P}-r_{+}$ diagrams) there is a van der Waals like phase transition for the obtained black hole solutions. The critical behavior is indicated as blue-bold line in the mentioned diagrams. As we expected the results of all diagrams are consistent. 

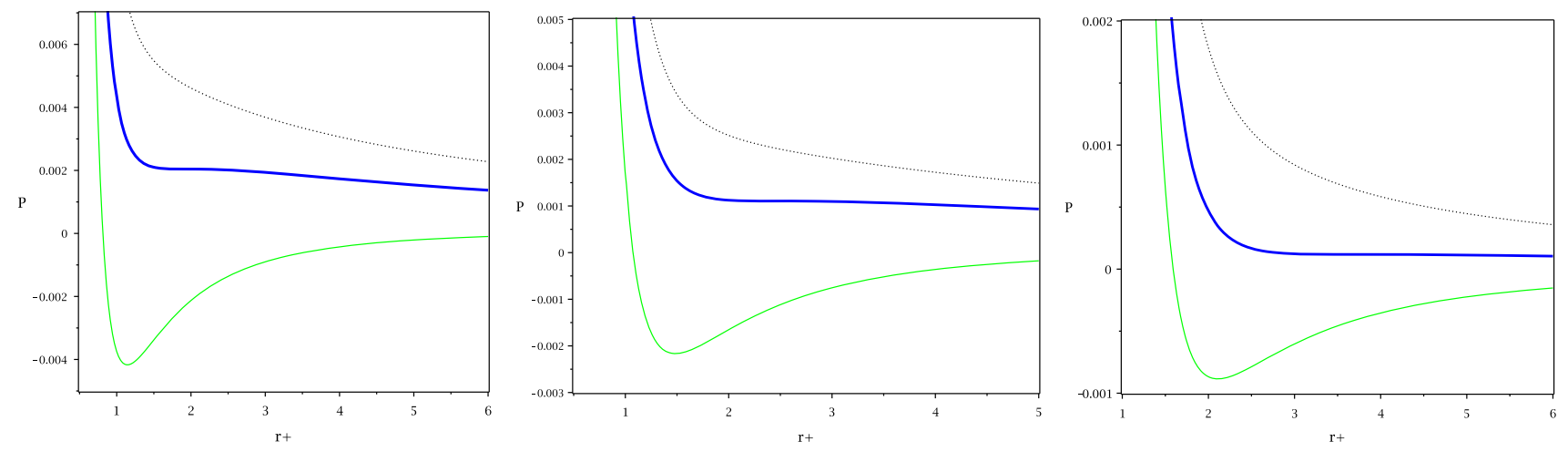

Fig. $2 P-r_{+}$diagrams for $C_{+}=-1, k=1, r_{0}=1, T<T_{c}$ (green continuous line), $T=T_{c}$ (blue bold line) and $T>T_{c}$ (black dashed line). "Note: $z=-0.1$ (left panel), $z=0$ (middle panel) and, $z=1$ (right panel)"
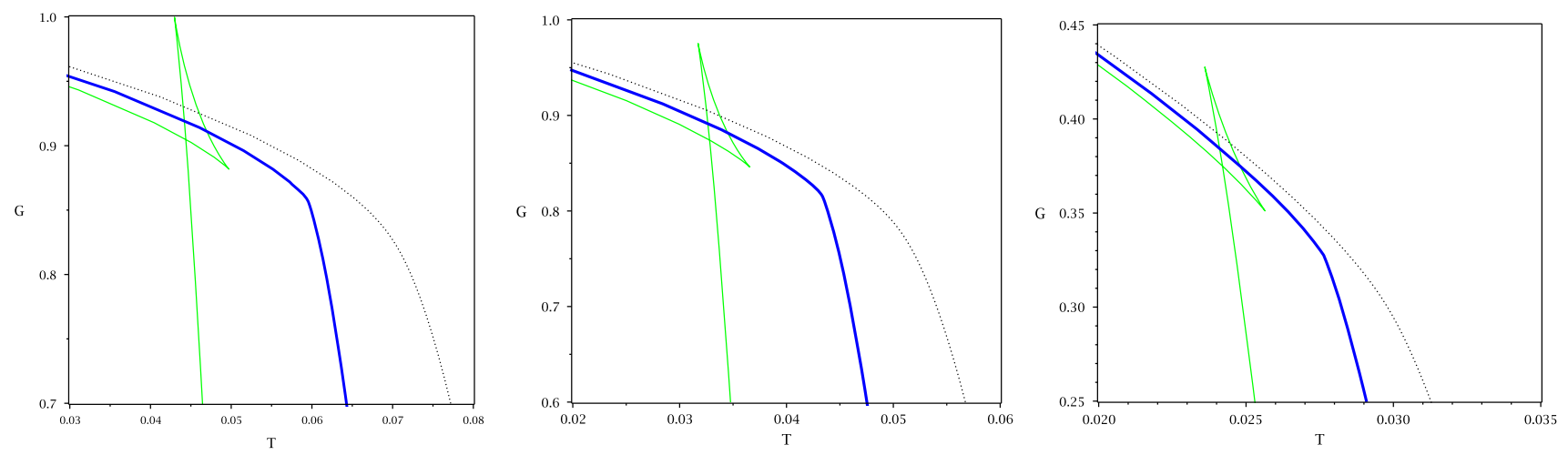

Fig. 3 Gibbs free energy versus temperature for $C_{+}=-1, k=1, r_{0}=1, P<P_{c}$ (green continuous line), $P=P_{c}$ (blue bold line) and $P>P_{c}$ (black dashed line). "Note: $z=-0.1$ (left panel), $z=0$ (middle panel) and $z=1$ (right panel)"
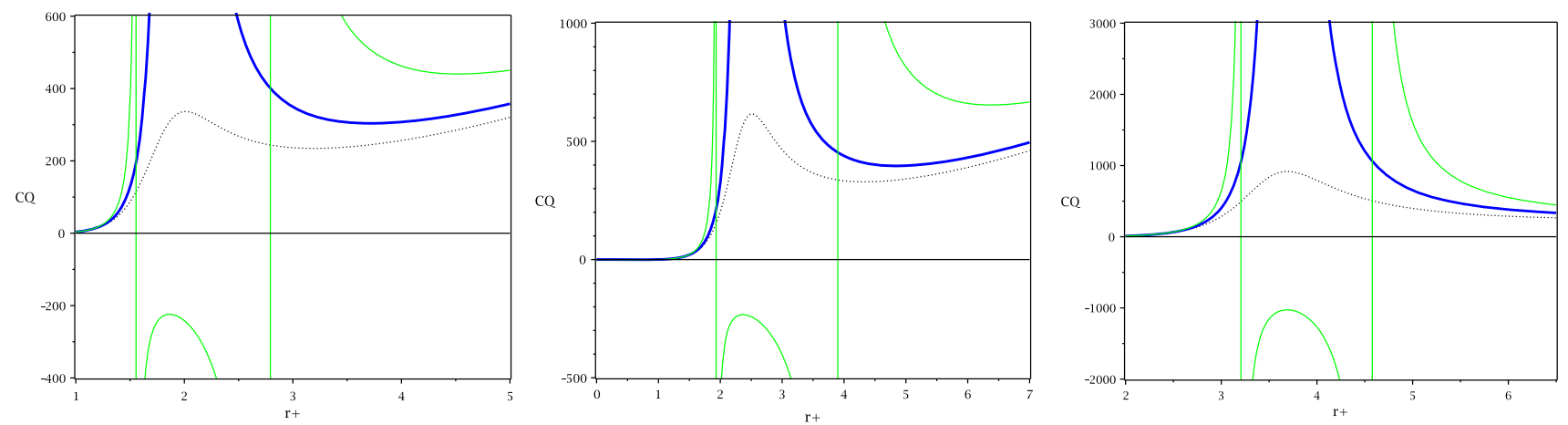

Fig. 4 Heat capacity versus $r_{+}$for $C_{+}=-1, k=1, r_{0}=1, P<P_{c}$ (green continuous line), $P=P_{c}$ (blue bold line) and $P>P_{c}$ (black dashed line). "Note: $z=-0.1$ (left panel), $z=0$ (middle panel) and $z=1$ (right panel)"

Strictly speaking, regarding isotherm $P-r_{+}$diagrams, we find that for $T>T_{C}$ an ideal gas behavior with no phase transition is observed. The critical isotherm is plotted for $T=T_{c}$ with an inflection point at $P=P_{c}, r_{+}=r_{c}$. For $T<T_{c}$ a van der Waals like shape is appeared.

Taking into account $G-T$ diagrams, one can find a smooth curve for $P>P_{c}$, a continuously curve which is not differentiable at a point with $P=P_{c}$ and $T=T_{c}$, and a swallow-tail shape for $P<P_{c}$, which is the characteristic of a phase transition in $G-T$ diagrams.
In addition, having a look at the heat capacity diagrams, we find that there is thermally stable black holes with positive definite heat capacity for $P>P_{c}$, while for the critical case, $P=P_{c}$, there is only one divergence point in the heat capacity diagrams, in which the positive sign of $C_{Q, P}$ does not change around the singularity. Finally for $P<P_{c}$, we observe two divergence points, in which the heat capacity is negative between them, and therefore, there is a phase transition between the mentioned two divergence points. 

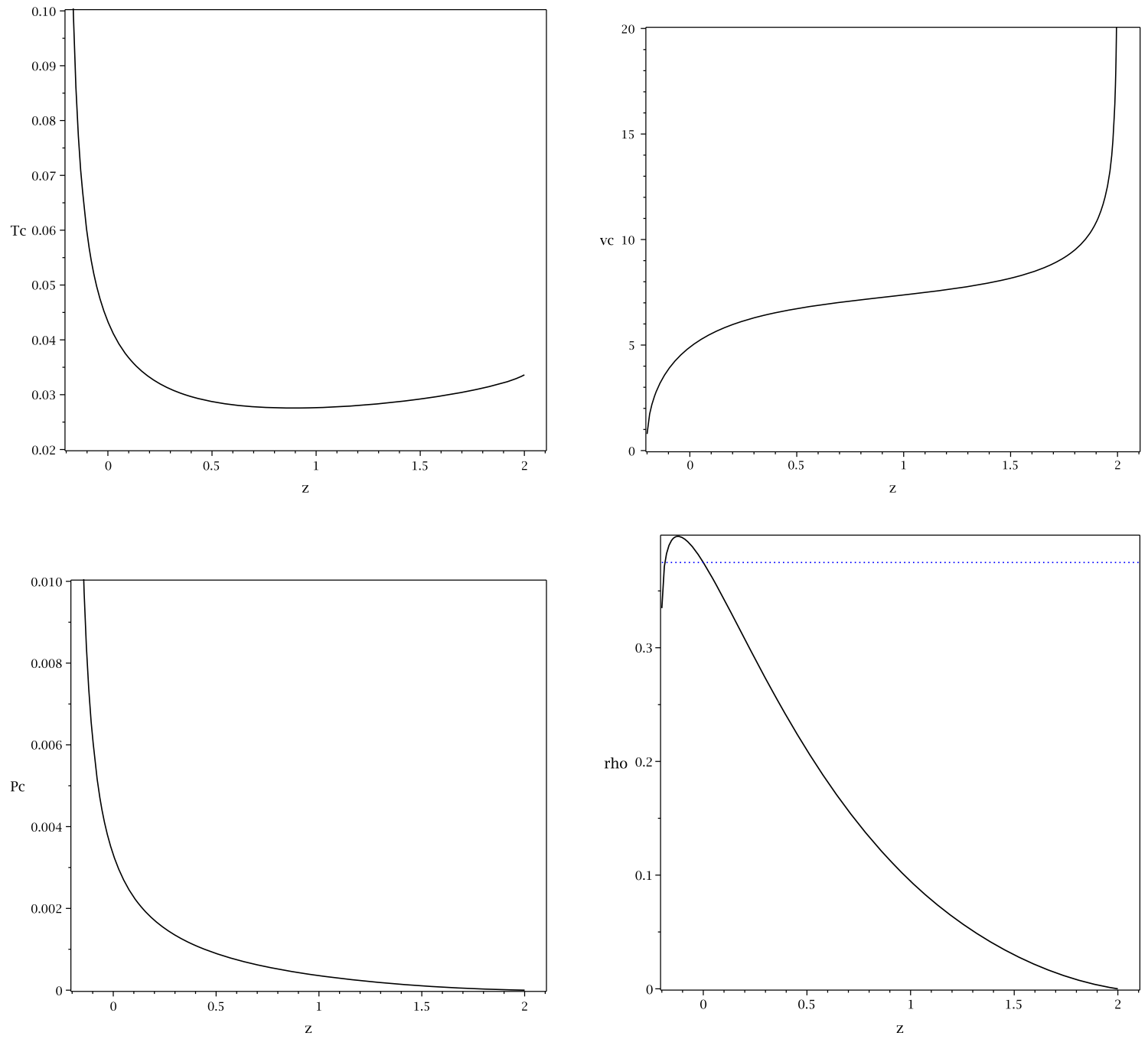

Fig. 5 Critical quantities versus $z$ for $C_{+}=-1, k=1$ and $r_{0}=1$. Left-up panel: $T_{c}$ versus $z$, right-up panel: $v_{c}$ versus $z$, left-down panel: $P_{c}$ versus $z$, right-down panel: black continuous line $\rho_{c}$ versus $z$ and blue dotted line is $\rho_{c}=\frac{3}{8}$

Table 1 Critical quantities for $k=1, r_{0}=1$ and $C_{+}=-1$

\begin{tabular}{lllll}
\hline $\mathrm{z}$ & $T_{c}$ & $v_{c}=2 r_{c}$ & $P_{c}$ & $\rho_{c}=\frac{P_{c} v_{c}}{T_{c}}$ \\
\hline-0.1 & 0.0595 & 3.85 & 0.0061 & 0.397 \\
0.0 & 0.043 & 4.90 & 0.0033 & 0.375 \\
0.1 & 0.036 & 5.53 & 0.0023 & 0.343 \\
0.5 & 0.028 & 6.72 & 0.0009 & 0.210 \\
1.0 & 0.027 & 7.37 & 0.0003 & 0.095 \\
1.5 & 0.029 & 8.17 & 0.0001 & 0.030 \\
\hline
\end{tabular}

Having a glance at the critical quantities, one can find different behaviors. According to Fig. 5 and Table 1, we find that $P_{c}\left(v_{c}\right)$ is a decreasing (an increasing) function of $z$, while there is a minimum (maximum) for $T_{c}\left(\rho_{c}\right)$. Therefore, in order to have a universal ratio such as the van der Waals fluid, one can obtain $z \simeq-0.1853$, in addition to $z=0$. It is also worth mentioning that for $z \longrightarrow 2^{-}$, both $P_{c}$ and $\rho_{c}$ vanish and criticality is disappeared. For $z>2$, all critical values are complex and one cannot obtain real valued critical quantities. All the mentioned calculations are done for the spherical horizon, $k=1$. Since some of the critical quantities depend on the topological factor $(k)$, linearly, one cannot find a nonzero critical quantity for black holes with flat horizon. But for hyperbolic horizon, one may look for the possible van der Waals like behavior, the same happens in massive gravity [48]. We postpone this interesting subject to appendix.

\section{Conclusion}

In this paper, we have studied thermodynamic behavior of topological black hole solutions with Lifshitz-like spacetime. We have worked in a special class of $F(R)$ gravity models with constant Ricci scalar in which satisfies two simultane- 
ous conditions, $F\left(R_{0}\right)=0$ and $F_{R}=0$. We have shown that although these solutions are asymptotically AdS with an effective cosmological constant for nonzero Ricci scalar, for vanishing Ricci scalar obtained solutions are not asymptotically flat unless for vanishing Lifshitz parameter.

We also calculated thermodynamic quantities and found that the solutions of this special class of modified gravity do not undergo the usual entropy and mass that reported in $F(R)$ gravity black holes, since $F_{R}=0$. We have calculated the entropy by using the first law of thermodynamics and found that it reduces to area law for vanishing Lifshitz parameter, $z=0$.

In addition, we investigate the phase transition in the extended phase space thermodynamics by considering the cosmological constant (which is proportional to the constant Ricci scalar) as a thermodynamical pressure for the spherical horizon black holes. We have studied the van der Waals like behavior by investigating three diagrams: isothermal pressure-volume, isobaric Gibbs free energy-temperature, and isobaric (isocharge) heat capacity-horizon radius. We have found that all the mentioned diagrams have consistent results to show three cases: completely stable state without any phase transition, critical behavior in the critical diagrams, and existence of a van der Waals like phase transition. We have shown that the Lifshitz parameter has an important role for the values of critical quantities.

We also extended our calculations to the case of hyperbolic horizon black holes and showed that a van der Waals like behavior can be observed only for negative temperature. In other words, for $k=-1$, although all physical quantities are positive, temperature is negative which may explain based on a quantum behavior of the black holes. Since it is a new result in the context of $F(R)$ gravity, it will be interesting to work on its nature by calculation of temperature based on statistical mechanics and simulate it with a quantum system with negative temperature.
Acknowledgements We are grateful to the anonymous referees for the insightful comments and suggestions, which have allowed us to improve this paper significantly. SHH wishes to thank Shiraz University Research Council. This work has been supported financially by the Research Institute for Astronomy and Astrophysics of Maragha, Iran.

Data Availability Statement This manuscript has no associated data or the data will not be deposited. [Authors' comment: This is a theoretical work and no experimental data were used.]

Open Access This article is distributed under the terms of the Creative Commons Attribution 4.0 International License (http://creativecomm ons.org/licenses/by/4.0/), which permits unrestricted use, distribution, and reproduction in any medium, provided you give appropriate credit to the original author(s) and the source, provide a link to the Creative Commons license, and indicate if changes were made.

Funded by SCOAP 3 .

\section{Appendix A: van der Waals like behavior for $k=-1$}

Here, we are going to examine the possible van der Waals like behavior for the black hole solutions with hyperbolic horizon $(k=-1)$. According to Eq. (33), Fig. 6 and Table 2, we find that a van der Waals behavior for hyperbolic horizon is observed. It is notable that although the critical volume and pressure are positive, the critical temperature is negative. In other words, all physical quantities such as Gibbs free energy, volume and pressure are positive in this case, but the same as those of nuclear spins system in an external magnetic field and population inversion in laser, temperature is negative. In this regard, although the swallowtail shape in $G-T$ diagram and van der Waals behavior in $P-r_{+}$figure make sense, the interpretation of phase transition (between two stable states) based on the (positivity of) heat capacity should change. Since negative temperature of physical system is a quantum mechanical behavior (it is not observed in classical systems), it will be interesting to investigate the thermodynamic behavior of the mentioned black hole solutions with the statistical mechanical approach.
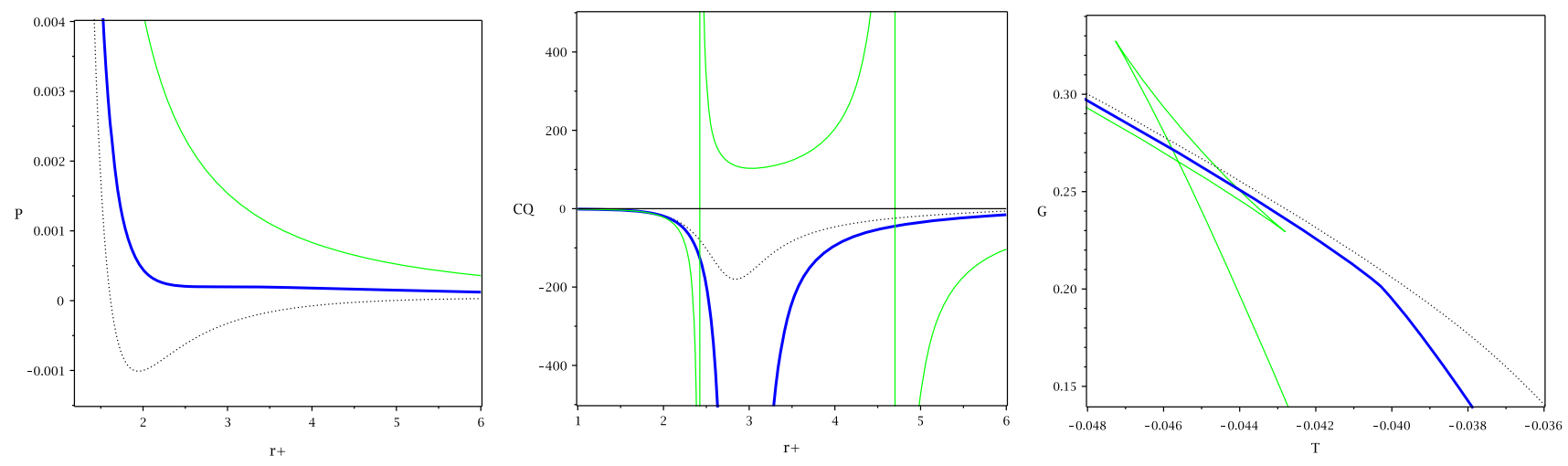

Fig. 6 Diagrams of $P-r_{+}$(left), $C_{Q, P}-r_{+}$(middle) and $G-T$ (right) for $C_{+}=-1, k=-1, r_{0}=1, z=3$ 
Table 2 critical quantities for $k=-1, r_{0}=1$ and $C_{+}=-1$

\begin{tabular}{lllll}
\hline $\mathrm{z}$ & $T_{c}$ & $v_{c}=2 r_{c}$ & $P_{c}$ & $\rho_{c}=\frac{P_{c} v_{c}}{T_{c}}$ \\
\hline 2.5 & -0.0382 & 3.48 & 0.00009 & -0.008 \\
3.0 & -0.0403 & 2.91 & 0.00020 & -0.014 \\
3.5 & -0.0417 & 2.61 & 0.00030 & -0.019 \\
4.0 & -0.0426 & 2.41 & 0.00038 & -0.022 \\
4.5 & -0.0433 & 2.27 & 0.00044 & -0.023 \\
5.0 & -0.0438 & 2.16 & 0.00049 & -0.024 \\
\hline
\end{tabular}

Table 3 QNMs $\left(\omega_{r}-i \omega_{i}\right)$ for $C_{+}=0.25, C_{-}=0.25, k=1, z=0$, $R_{0}=1$, and $r_{0}=1$

\begin{tabular}{llll}
\hline$n$ & $l=1$ & $l=2$ & $l=3$ \\
\hline 0 & $0.9514-0.4385 i$ & $1.622-0.4218 i$ & $2.281-0.4173 i$ \\
1 & - & $1.510-1.294 i$ & $2.197-1.267 i$ \\
2 & - & - & $2.051-2.144 i$ \\
\hline
\end{tabular}

\section{Appendix B: Dynamical stability}

Here, we consider a massless scalar perturbation in the background of the black hole spacetime and obtain the quasinormal frequencies (QNFs) by using the third order WKB approximation. The WKB approximation was first applied to the problem of scattering around black holes [68], and then extended to the third order [69]. This method can be used for an effective potential that forms a barrier potential and takes constant values at the event horizon and cosmological horizon. In addition, we concentrate our attention to the fixed values of free parameters of metric function throughout the text.

The equation of motion for a massless scalar field is given by

$\square \Phi=0$

If we consider modes as

$\Phi(t, r, \theta, \varphi)=\frac{1}{r} \Psi(r) Y_{l, m}(\theta, \varphi) e^{-i \omega t}$,

where $Y_{l, m}(\theta, \varphi)$ is the spherical harmonics, the equation of motion (38) reduces to the following wave equation

$\left[\partial_{x}^{2}+\omega^{2}-V_{l}(x)\right] \Psi_{l}(x)=0$,

where $x$ is the tortoise coordinate

$d x=\frac{d r}{e^{\alpha(r)} B(r)}$,

and the effective potential $V_{l}(x)$ is given by

$V_{l}(x)=e^{2 \alpha(r)} B(r)\left[\frac{l(l+1)}{r^{2}}+\frac{B(r) \alpha^{\prime}(r)}{r}+\frac{B^{\prime}(r)}{r}\right]$,

where $l$ is the angular quantum number. One can obtain the QNMs of this perturbation by considering proper boundary conditions as follows

Table 4 The fundamental

QNMs $\left(\omega_{r}-i \omega_{i}\right)$ for

$C_{+}=0.25, C_{-}=0.25, k=1$,

$R_{0}=1$, and $r_{0}=1$

\begin{tabular}{llll}
\hline$z$ & $l=1$ & $l=2$ & $l=3$ \\
\hline-0.1 & $1.035-0.4775 i$ & $1.758-0.4598 i$ & $2.471-0.4553 i$ \\
0.5 & $0.6881-0.2859 i$ & $1.180-0.2733 i$ & $1.661-0.2696 i$ \\
1.0 & $0.5409-0.1941 i$ & $0.9247-0.1827 i$ & $1.304-0.1797 i$ \\
1.5 & $0.4295-0.1361 i$ & $0.7396-0.1285 i$ & $1.045-0.1268 i$ \\
2.0 & $0.3247-0.09380 i$ & $0.5670-0.09095 i$ & $0.8038-0.09034 i$ \\
2.5 & $0.1978-0.05376 i$ & $0.3490-0.05331 i$ & $0.4959-0.05322 i$ \\
\hline
\end{tabular}
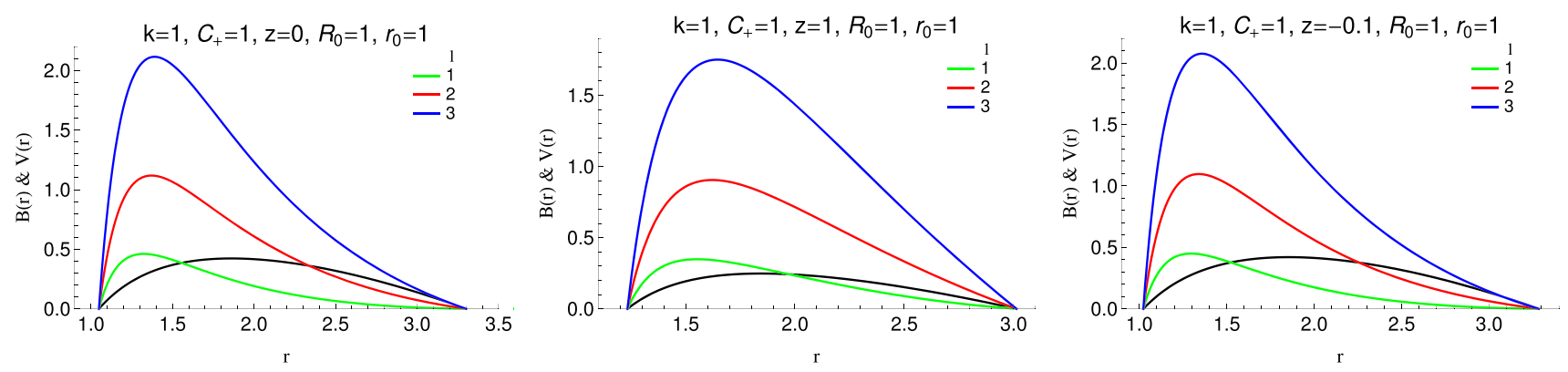

Fig. 7 The effective potential (colored lines) and metric function (black line) versus the radial coordinate $r$ 


$$
\begin{array}{llrl}
\Psi_{l}(r) \sim e^{-i \omega x} & \text { as } & x \rightarrow-\infty\left(r \rightarrow r_{e}\right), \\
\Psi_{l}(r) \sim e^{i \omega x} & \text { as } & x \rightarrow \infty\left(r \rightarrow r_{c}\right),
\end{array}
$$

which means that no wave comes from the event horizon and cosmological horizon ( $r_{e}$ is the event horizon and $r_{c}$ is the cosmological horizon). We should consider theses boundary conditions to obtain the QNFs.

The third order WKB formula is given by

$$
\frac{i\left(\omega^{2}-V_{0}\right)}{\sqrt{-2 V_{0}^{\prime \prime}}}-\Lambda_{2}-\Lambda_{3}=n+\frac{1}{2} ; \quad n=0,1,2, \ldots
$$

where $V_{0}$ is the value of effective potential at its local maximum, $n$ is the overtone number, and the correction terms $\Lambda_{2}$ and $\Lambda_{3}$ are given in [69]. The results are given in the Tables 3 and 4. The real (imaginary) part of the frequencies decreases (increases) as the overtone number increases, but the angular quantum number has opposite behavior. On the other hand, increasing in $z$ leads to decreasing both the real and imaginary parts of the frequencies.

In the case of dynamical stability, the Fig. 7 shows the behavior of the effective potential in which this potential forms a barrier potential. Since the effective potentials are positive everywhere, then $\int_{-\infty}^{+\infty} V_{l}(x) d x>0$ and the obtained black hole solutions are dynamically stable under massless scalar perturbations [70]. Therefore, it is possible to find the dynamically stable black holes by using the obtained black hole solutions.

\section{Appendix C: Non-constant Ricci scalar}

Now, we give a discussion regarding an arbitrary function of Ricci scalar $(R=R(r))$. Considering the metric ansatz (7) with an arbitrary Ricci scalar, one finds the following exact solutions

$B(r)=K-\frac{C_{ \pm}}{r^{b_{ \pm}}} \pm \frac{\left(2+b_{-}\right)\left(2+b_{+}\right)}{\left(b_{+}-b_{-}\right) r^{b_{ \pm}}} \int r^{1+b_{ \pm}} \lambda(r) d r$

where $K$ and $b_{ \pm}$are introduced before, and following Eq. (11), we define $\lambda(r)=\frac{2 R(r)}{z^{2}+8 z+24}$. It is straightforward to show that Eq. (45) reduces to (9) for constant Ricci scalar, $\lambda(r)=\lambda$.

In order to obtain the temperature, we follow Eq. (19). After some manipulations, we obtain

$$
\begin{aligned}
T= & \left\{\frac{K b_{-} r_{+}^{b_{+}}-C_{+}\left(b_{+}-b_{-}\right)-\left(2+b_{+}\right)\left(2+b_{-}\right) \Theta_{+}}{4 \pi r_{+}^{1+b_{+}}}\right\} \\
& \left(\frac{r_{+}}{r_{0}}\right)^{\frac{z}{2}},
\end{aligned}
$$

in which $\Theta_{+}=\int^{r=r_{+}} r^{1+b_{+}} \lambda(r) d r$.
As we mentioned before, we have to calculate the entropy via the first law of thermodynamics, (23). As one expects, the new relation of entropy is the same as that of reported in Eq. (24).

Now, one may looking for possible phase transition. To do so, we have to specify the functional form of the Ricci scalar. Specifying the Ricci scalar, one can define a suitable dynamical pressure and investigate critical behavior of the solutions. Since it is straightforward, we abandon the presentation of a specific example, for the sake of brevity.

\section{References}

1. G. Cognola, E. Elizalde, S. Nojiri, S.D. Odintsov, S. Zerbini, JCAP 0502, 25 (2005)

2. A. Cooney, S. DeDeo, D. Psaltis, Phys. Rev. D 82, 064033 (2010)

3. J.Q. Guo, A.V. Frolov, Phys. Rev. D 88, 124036 (2013)

4. T.P. Sotiriou, V. Faraoni, Rev. Mod. Phys. 82, 451 (2010)

5. A. Palatini, Rend. Circ. Mat. Palermo. 43, 203 (1919)

6. L. Amendola, R. Gannouji, D. Polarski, S. Tsujikawa, Phys. Rev. D 75, 083504 (2007)

7. B. Li, J.D. Barrow, Phys. Rev. D 75, 083502 (2007)

8. W. Hu, I. Sawicki, Phys. Rev. D 76, 104043 (2007)

9. A.A. Starobinsky, J. Exp. Theor. Phys. Lett. 86, 157 (2007)

10. S. Capozziello, S.J.G. Gionti, D. Vernieri, JCAP 1601, 015 (2016)

11. S.S.H. Hendi, R.B. Mann, N. Riazi, B.Eslam Panah, Phys. Rev. D 86, 104034 (2012)

12. S.H. Hendi, Phys. Lett. B 690, 220 (2010)

13. S. Capozziello, M.D. Laurentis, Phys. Rep. 509, 31422 (2015)

14. K.I. Maeda, Phys. Rev. D 39, 3159 (1989)

15. J. Kluson, JHEP 11, 078 (2009)

16. M. Chaichian, S. Nojiri, S.D. Odintsov, M. Oksanen, A. Tureanu, Class. Quantum Gravity 27, 185021 (2010)

17. S. Carloni, M. Chaichian, S. Nojiri, S.D. Odintsov, M. Oksanen, A. Tureanu, Phys. Rev. D 82, 065020 (2010)

18. J. Kluson, Phys. Rev. D 82, 044004 (2010)

19. J. Kluson, S. Nojiri, S.D. Odintsov, D. Saez-Gomez, Eur. Phys. J. C 71, 1690 (2011)

20. J. Kluson, Phys. Rev. D 84, 104014 (2011)

21. S. Nojiri, S.D. Odintsov, Phys. Rep. 505, 59 (2011)

22. S. Chattopadhyay, R. Ghosh, Astrophys. Space Sci. 341, 669 (2012)

23. A.J. Lopez-Revelles, R. Myrzakulov, D. Saez-Gomez, Phys. Rev. D 85, 103521 (2012)

24. J.Q. Guo, D. Wang, A.V. Frolov, Phys. Rev. D 90, 024017 (2014)

25. P. Horava, Phys. Rev. D 79, 084008 (2009)

26. J.F. Donoghue, Phys. Rev. D 50, 3874 (1994)

27. S.W. Hawking, Commun. Math. Phys. 43, 199 (1975)

28. S.W. Hawking, Nature 248, 30 (1974)

29. A. Strominger, C. Vafa, Phys. Lett. B 379, 99 (1996)

30. C. Rovelli, Phys. Rev. Lett. 77, 3288 (1996)

31. A. Strominger, JHEP 9802, 009 (1998)

32. G. 't Hooft. arXiv:gr-qc/9310026

33. L. Susskind, J. Math. Phys. 36, 6377 (1995)

34. J.M. Maldacena, Adv. Theor. Math. Phys. 2, 231 (1998)

35. O. Aharony, S.S. Gubser, J.M. Maldacena, H. Ooguri, Y. Oz, Phys. Rep. 323, 183 (2000)

36. E. Witten, Adv. Theor. Math. Phys. 2, 253 (1998)

37. B.P. Dolan, Class. Quantum Gravity 28, 125020 (2011)

38. D. Kubiznak, R.B. Mann, J. High Energy Phys. 1207, 033 (2012)

39. N. Altamirano, D. Kubiznak, R.B. Mann, Z. Sherkatghanad, Class. Quantum Gravity 31, 042001 (2014) 
40. S.W. Wei, Y.X. Liu, Phys. Rev. D 90, 044057 (2014)

41. R.A. Hennigar, W.G. Brenna, R.B. Mann, JHEP 1507, 077 (2015)

42. S. Gunasekaran, R.B. Mann, D. Kubiznak, JHEP 1211, 110 (2012)

43. A.M. Frassino, D. Kubiznak, R.B. Mann, F. Simovic, JHEP 1409, $080(2014)$

44. R.A. Hennigar, R.B. Mann, Entropy 17, 8056 (2015)

45. C.V. Johnson, Class. Quantum Gravity 31, 205002 (2014)

46. A. Belhaj, M. Chabab, H. El Moumni, K. Masmar, M.B. Sedra, A. Segui, JHEP 1505, 149 (2015)

47. S.H. Hendi, Z.S. Taghadomi, C. Corda, Phys. Rev. D 97, 084039 (2018)

48. S.H. Hendi, R.B. Mann, S. Panahiyan, B. Eslam Panah, Phys. Rev. D 95, 021501(R) (2017)

49. E.M. Purcell, R.V. Pound, Phys. Rev. 81, 279 (1951)

50. N.F. Ramsey, Phys. Rev. 103, 20 (1956)

51. M.J. Klein, Phys. Rev. 104, 589 (1956)

52. P. Varga, Phys. Rev. E. 57, 6487 (1998)

53. W. Hsu, R. Barakat, Phys. Rev. B 46, 6760 (1992)

54. M. Akbar, R.G. Cai, Phys. Lett. B 648, 243 (2007)
55. K. Bamba, C.Q. Geng, Phys. Lett. B 679, 282 (2009)

56. J. Man, H. Cheng, Phys. Rev. D 87, 044002 (2013)

57. Y.G. Miao, F.F. Yuan, Z.Z. Zhang, Int. J. Mod. Phys. D 23, 1450093 (2014)

58. B. Jawad, S. Rani, D. Momeni, F. Gulshan, R. Myrzakulov. arXiv: 1602.06252

59. J.X. Mo, G.Q. Li, X.B. Xu, Eur. Phys. J. C 76, 545 (2016)

60. S. Soroushfar, R. Saffari, N. Kamvar, Eur. Phys. J. C 76, 476 (2016)

61. Y. Zheng, R.J. Yang, Eur. Phys. J. C 78, 682 (2018)

62. X. Fang, X. He, J. Jing, Eur. Phys. J. C 78, 623 (2018)

63. M. Calza, M. Rinaldi, L. Sebastiani, Eur. Phys. J. C 78, 178 (2018)

64. C.Q. Geng, W.C. Hsu, J.R. Lu, L.W. Luo, Entropy 21, 172 (2019)

65. G. Gibbons, R. Kallosh, B. Kol, Phys. Rev. Lett. 77, 4992 (1996)

66. J.D. Brown, C. Teitelboim, Phys. Lett. B 195, 177 (1987)

67. M.M. Caldarelli, G. Cognola, D. Klemm, Class. Quantum Gravity 17, 399 (2000)

68. B.F. Schutz, C.M. Will, Astrophys. J. Lett. 291, L33 (1985)

69. S. Iyer, C.M. Will, Phys. Rev. D 35, 3621 (1987)

70. B. Simon, Ann. Phys. 97, 279 (1976) 